\title{
Bilateral Synchronous Adrenal Tumors in Lynch Syndrome
}

\author{
Mason Vierra ${ }^{1 *}$ and Arina Ganeles, $M D^{2}$ \\ ${ }^{1}$ University of Chicago Pritzker School of Medicine, USA \\ ${ }^{2}$ Community Hospital of the Monterey Peninsula, USA
}

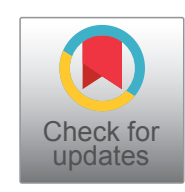

\begin{abstract}
A 65-year-old Hispanic male with Lynch Syndrome and a history colon cancer was incidentally found to have bilateral adrenal tumors by CT scan. A PET scan revealed marked FDG avidity of both adrenal masses; with an SUV of up to 24.5. The patient was asymptomatic at the time. The patient underwent bilateral adrenalectomy and left nephrectomy. Final pathology demonstrated high-grade malignant spindle cell neoplasm with sarcomatoid features. Immunohistochemistry for the MMR proteins showed decreased protein expression of $M L H 1$, negative staining for PMS2, and intact tumor staining of MSH2 and MSH6. Nine months postoperatively he is clinically well, on oral hormone replacement without recurrent or residual tumor. Adrenal cortical carcinoma is a rare tumor but patients with Lynch Syndrome may have an increased risk of developing adrenal malignancies.
\end{abstract}

\section{Keywords}

Lynch syndrome, Adrenal cortical carcinoma, Mismatch repair, Adrenalectomy

\section{Introduction}

Lynch syndrome (LS) is one of the most common hereditary cancer syndromes, occurring in as many as 1 in 279 people in the U.S. and exhibiting an autosomal dominant inheritance pattern [1]. Linked to mutations in genes involved with DNA mismatch repair, LS is associated with a significantly elevated risk of colorectal and endometrial cancer most commonly, as well as several other cancers. While the cancer spectrum associated with LS has not traditionally included sarcomas, several cases of sarcomas arising in LS patients have been reported.

Cancers of the adrenal glands are very rare. When they do arise, they are typically adrenocortical carcinomas (ACC), and very infrequently present as sarcomas. ACC has been associated with LS, but research is limited to just 13 case studies and one small prospective series. There are no reports of other adrenal gland cancer types in Lynch syndrome.

Here we report a case of a patient with Lynch syndrome with bilateral, synchronous adrenal cancers of sarcomatoid features.

\section{Case Report}

A 65-year-old Hispanic male with a pathogenic variant in MLH1 and a history of two prior colon cancers was incidentally found to have bilateral adrenal malignant sarcomatoid tumors on abdominal imaging.

In November of 2003 , the patient presented with a perforated distal colon cancer for which he underwent surgery.
Pathology demonstrated a Duke $B$, node negative cancer, but, because of the perforation, he was started on adjuvant capecitabine and oxaliplatin. He developed GI bleeding after two cycles, and further workup revealed a cancer of the right colon, for which he underwent resection in May of 2004. His surgical course from these operations was complicated and in total required 5 laparotomies. He subsequently received 6 months of capecitabine and oxaliplatin.

The patient was followed closely as an outpatient and was disease free until 2009, when he developed elevated RBC mass. A workup for polycythemia vera was negative for a JAK2 mutation but he was found to have a small lower pole left kidney lesion and hematuria. In 2014 this lesion was noted to be $1.6 \mathrm{~cm}$.

In February 2017 the patient underwent multi-gene panel testing and was shown to have a pathogenic variant in the MLH1 gene, consistent with Lynch Syndrome. Family history was unobtainable.

*Corresponding author: Mason Vierra, University of Chicago Pritzker School of Medicine, 975 Mesa Road, Monterey, California 93940, USA, Tel: 831-402-7567

Accepted: August 07, 2021

Published online: August 09, 2021

Citation: Vierra M, Ganeles A (2021) Bilateral Synchronous Adrenal Tumors in Lynch Syndrome. J Surgical Endocrinol 3(2):94-96 
In March of 2018, the patient's left renal lesion had grown to $1.7 \mathrm{~cm}$ on follow-up CT scan and was characterized as a cyst. In November of 2019, a CT scan performed to follow up on the renal cyst noted a new $6.9 \mathrm{~cm}$ left adrenal tumor. In December 2019, a CT scan with contrast demonstrated bilateral adrenal masses, $7.6 \mathrm{~cm}$ on the left and $2.9 \mathrm{~cm}$ on the right (Figure 1). In January 2020 a PET scan revealed marked FDG avidity of both adrenal masses, with an SUV of up to 24.5 (Figure 2). The patient was asymptomatic at the time with ECOG performance status of 0 .

In March of 2020, the patient underwent bilateral adrenalectomy. It was necessary to remove the left kidney, as it was inseparable from the tumor. Final pathology demonstrated "high-grade malignant spindle cell neoplasm with sarcomatoid features." Immunohistochemistry for the MMR proteins showed decreased protein expression of

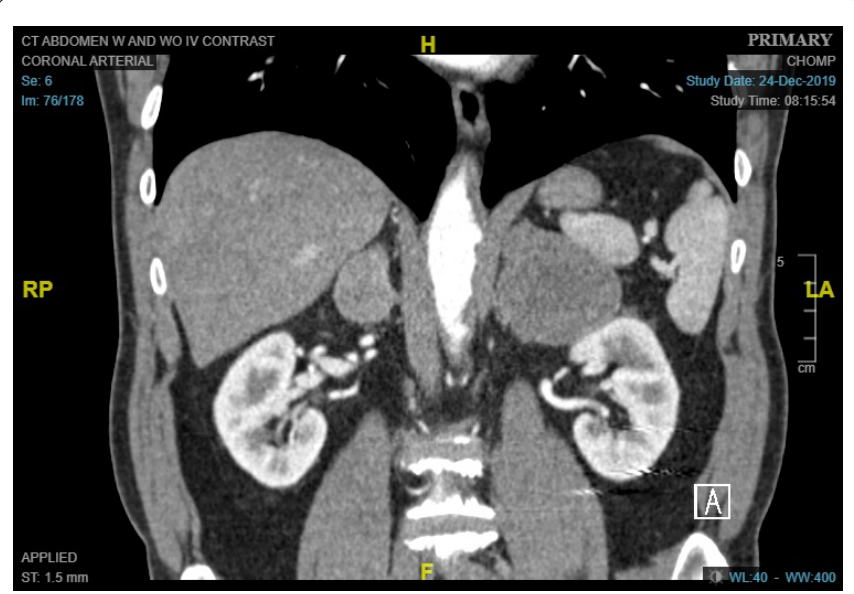

Figure 1: CT scan demonstrating bilateral adrenal masses, $7.6 \mathrm{~cm}$ on the left and $2.9 \mathrm{~cm}$ on the right.

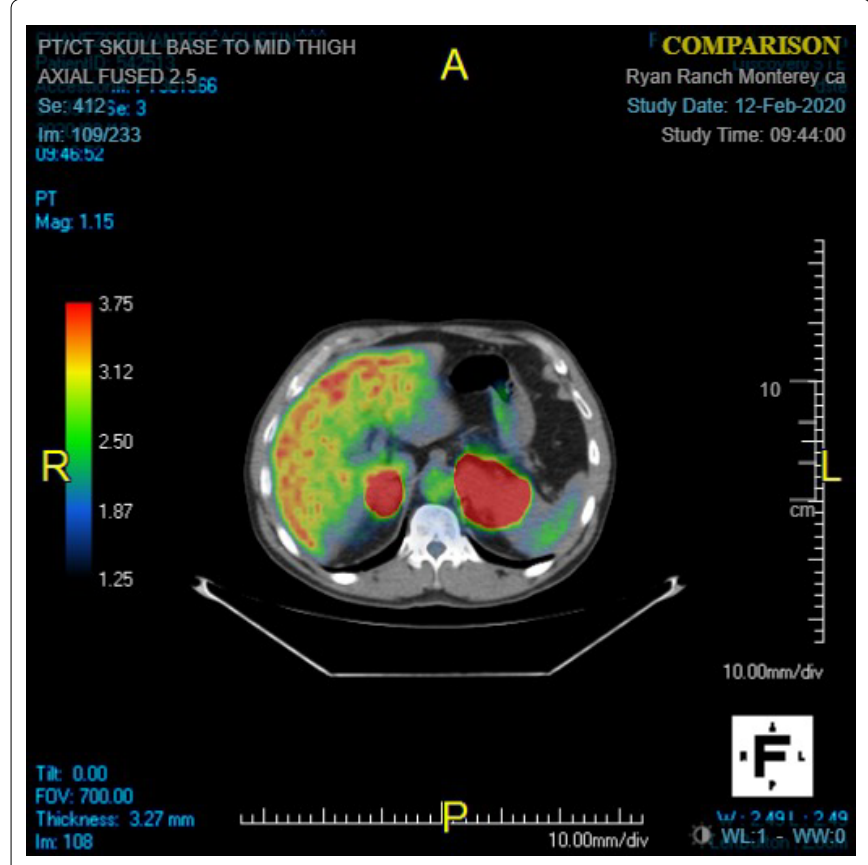

Figure 2: PET scan demonstrating FDG avidity of adrenal masses with an SUV of up to 24.5 .

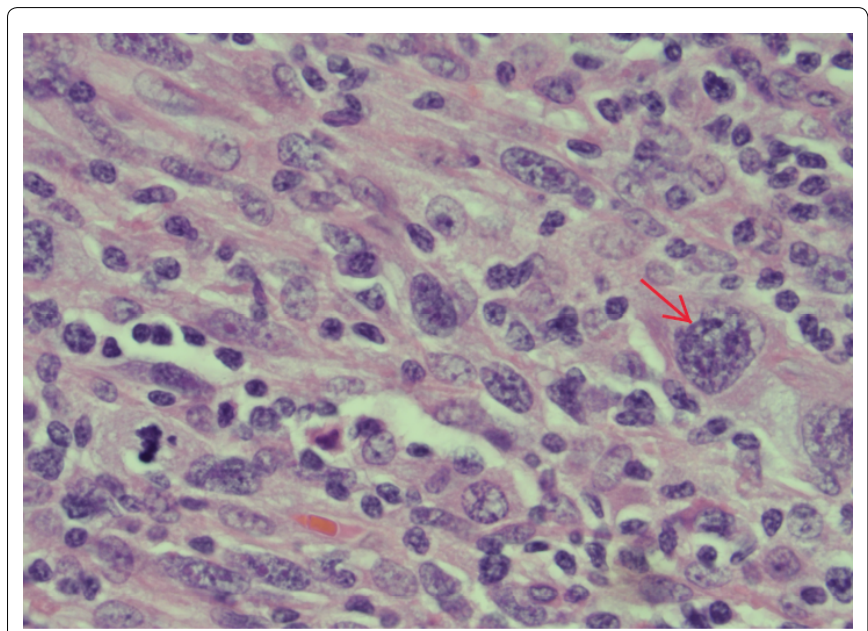

Figure 3: Histology slide of adrenal tumor; red arrow points to a cell with spindled morphology with marked nuclear pleomorphism, nuclear enlargement and irregular nuclear membranes, and with abundant eosinophilic cytoplasm.

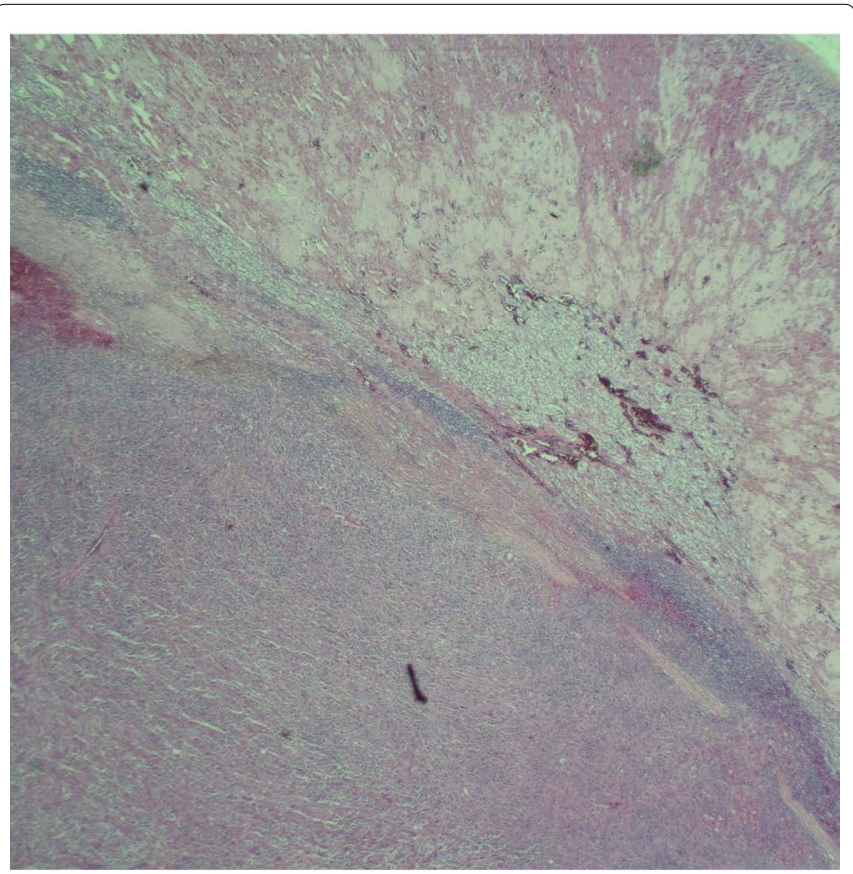

Figure 4: Histology demonstrating adipose tissue, a thin rim of remaining adrenal gland, and central tumor.

$M L H 1$, negative staining for $P M S 2$, and intact tumor staining of MSH2 and MSH6. S100 was negative. A Ki-67 stain showed a tumor proliferation of $50 \%$. The tumor proportion score (TPS SCORE) showed $90 \%$ positivity and was interpreted as showing high PD-L1 expression (Figure 3, Figure 4).

At 9 months postoperatively he is clinically well, maintained on oral hormone replacement and with no recurrent or residual tumor.

\section{Discussion}

We present here the case of a 65-year-old man with Lynch Syndrome due to a germline $M L H 1$ pathogenic variant and two previous colon cancers who was found incidentally with bilateral adrenal sarcomatoid cancers. To our knowledge, 
this is the first reported case of bilateral adrenal sarcomatoid cancers in a patient with LS.

While the link between LS and various types of cancer, especially colorectal and endometrial, is well established, the association between LS and adrenal cancers has been less well documented. For example, the association between LS and adrenal cortical carcinoma (ACC), the most common type of adrenal cancer, has been reported in only 13 case reports and a small case series. The case series found that $3.2 \%$ [2] of patients with ACC had LS, which is similar to the prevalence of LS seen in patients with colorectal (2-5\%) [35] and endometrial cancers (3\%) [6], and is higher than the prevalence of LS seen in the general population (0.35\%) [1].

The present case is unique for several reasons. First, the adrenal masses were spindle cell neoplasms with sarcomatoid features, as opposed to the more common ACC. Second, the tumors were large, bilateral, and synchronous without metastasis. Finally, immunohistochemistry demonstrated loss of MLH1 and PMS2 protein expression, with normal expression of MSH2 and MSH6, suggesting that this could have arisen through a mismatch repair deficiency pathway. Of the 13 cases previously referenced describing the association between LS and ACC, only one case had a MLH1 deficiency, the rest being $\mathrm{MSH} 2$ or $\mathrm{MSH} 6$ deficiencies [7].

The incidence of adrenal cancers, especially those of the sarcomatoid type presented here, is low enough that surveillance is not typically recommended in LS patients. However, the existing literature on the association between LS and adrenal cancers suggests that physicians should be aware of the possibility that they may be linked. Conversely, it could be argued that patients presenting with ACC and possibly other adrenal cancers should have their personal and family histories reviewed and consider germ-line testing. Microsatellite instability testing and immunohistochemistry testing could be applied to adrenal tumors to test for LS.

It is furthermore important to consider that the presence of LS in patients with adrenal cancers may have important implications for treatment. Immunotherapy has in recent years produced promising results in treating patients with LS-associated tumors and other tumors that demonstrate mismatch repair deficiency. Its use in patients with adrenal cancers has also been documented in a handful of trials, with mixed results $[8,9]$. However, these trials did not specifically differentiate patients based on a diagnosis of Lynch Syndrome, and immunotherapy in patients with both adrenal cancers and LS has only been described in a handful of case studies $[10,11]$. Much more research must be done to evaluate whether immunotherapy is as effective in treating LS-associated adrenal cancers as it has been in treating other cancers. Finally, sarcomas have historically not been associated with LS, although the association has been described in a handful of case reports. Thus, while this patient's presentation is quite rare and unique, further research should be done to determine whether LS is in fact a risk factor for sarcoma.

\section{Conclusion}

We describe here the unusual case of a man with Lynch Syndrome and two metachronous colon cancers who presented with bilateral adrenal cancers with sarcomatoid features. The patient underwent a bilateral adrenalectomy and a left nephrectomy. Though this is certainly a rare case, it is important to note that:

1. One 2013 prospective study found that the incidence of LS in patients who have ACC is similar to the incidence of LS among patients who present with colon cancer. It might be appropriate to consider genetic testing in patients who present with adrenal cancers of any kind.

2. Furthermore, patients with Lynch syndrome may have an increased risk of adrenal cancers that is sufficiently high to warrant screening.

3. Immunotherapy treatment of Lynch Syndrome cancers has shown to be effective, but its use in Lynch Syndrome patients with adrenal cancers hasn't been adequately studied.

4. While LS has not historically been associated with sarcomas, this case can be added to the existing literature linking the two. Perhaps this association should be studied in more depth.

\section{Disclosures}

None

\section{References}

1. Win AK, Jenkins MA, Dowty JG, et al. (2017) Prevalence and penetrance of major genes and polygenes for colorectal cancer. Cancer Epidemiol Biomarkers Prev 26: 404-412.

2. Raymond VM, Everett JN, Furtado LV, et al. (2013) Adrenocortical carcinoma is a lynch syndrome-associated cancer. J Clin Oncol 31: 3012-3018.

3. Barnetson RA, Tenesa A, Farrington SM, et al. (2006) Identification and survival of carriers of mutations in dna mismatch-repair genes in colon cancer. N Engl J Med 354: 2751-2763.

4. Hampel H, Frankel WL, Martin E, et al. (2008) Feasibility of screening for lynch syndrome among patients with colorectal cancer. J Clin Oncol 26: 5783-5788.

5. Yurgelun MB, Kulke MH, Fuchs CS, et al. (2017) Cancer susceptibility gene mutations in individuals with colorectal cancer. J Clin Oncol 35: 1086-1095.

6. Ryan NAJ, Glaire MA, Blake D, et al. (2019) The proportion of endometrial cancers associated with Lynch syndrome: A systematic review of the literature and meta-analysis. Genet Med 21: 2167-2180.

7. Kaur RJ, Pichurin PN, Hines JM, et al. (2019) Adrenal cortical carcinoma associated with lynch syndrome: A case report and review of literature. J Endocr Soc 3: 784-790.

8. Cosentini D, Grisanti S, Dalla Volta A, et al. (2018) Immunotherapy failure in adrenocortical cancer: Where next? Endocr Connect 7: E5-E8.

9. Raj N, Zheng Y, Kelly V, et al. (2020) PD-1 blockade in advanced adrenocortical carcinoma. J Clin Oncol 38: 71-80.

10. Head L, Kiseljak-Vassiliades K, Clark TJ, et al. (2019) Response to immunotherapy in combination with mitotane in patients with metastatic adrenocortical cancer. J Endocr Soc 3: 2295-2304.

11. Mota JM, Sousa LG, Braghiroli MI, et al. (2018) Pembrolizumab for metastatic adrenocortical carcinoma with high mutational burden: Two case reports. Medicine (Baltimore) 97: e13517. 